\title{
Twin boundary interactions with grain boundaries investigated in pure rhenium
}

\author{
Josh Kacher $^{1 *}$ and Andrew M. Minor ${ }^{1}$
}

${ }^{1}$ Department of Materials Science and Engineering, University of California, Berkeley, CA USA, and National Center for Electron Microscopy, Lawrence Berkeley National Laboratory, Berkeley, CA USA

*corresponding author: Josh Kacher <jkacherbyu@gmail.com>

\begin{abstract}
The mechanical behavior of pure rhenium was investigated using uniaxial compression tests, transmission electron microscopy, and electron backscatter diffraction characterization. The plasticity was characterized by a large amount of twin formation and propagation, including twin transmission across grain boundaries. In-depth analysis of the interactions of $\{1121\}<1126>$ twins with grain boundaries found that grain boundaries with misorientation angles below $\sim 25^{\circ}$ allowed twin transmission while grain boundaries with higher angles did not. Similar to dislocation interactions with grain boundaries, twin transmission was largely dictated by the minimization of the angle between the shear vectors of the incoming and outgoing twins.
\end{abstract}

Keywords: Twinning; rhenium; mechanical behavior; electron backscatter diffraction; transmission electron microscopy

\section{Introduction}

Due to the restricted number of slip planes, deformation in many hexagonal close-packed (HCP) metals involves twinning mechanisms. Consequentially, the mechanical behavior of HCP metals can be largely governed by the interaction of twins with defects. For example, interfaces such as grain boundaries can act as nucleation sites for twins as well as obstacles to twin propagation. An understanding of how these interactions unfold, including the dominant factors dictating their progression, is essential to a fundamental understanding of the mechanical behavior of HCP materials in application.

Re is unusual among HCP materials in that the dominant twining system, when either tensile or compressive stresses are imposed on the sample, is the $\{1121\}<1126\rangle[1,2]$. It is also unusual among refractory metals in that it has no known ductile to brittle transition. For this reason, as well as its excellent ductility and formability, high temperature strength, and creep resistance, it is an attractive material in extreme environment applications [3, 4]. However, limited availability and difficulty of extracting Re has limited both the application as well as the knowledge of Re. Characterization of the deformed microstructure of Re has been limited primarily to optical microscopy $[3,4]$ as well as a limited number of transmission electron microscopy (TEM) studies $[1,2,5]$. These studies reported observations of dislocation plasticity occurring on the basal and prismatic planes, as well as twinning activity on $\{1012\},\{1122\}$, and $\{1121\}$. 
Statistical studies using electron backscatter diffraction (EBSD) have investigated the behavior of twinning in deformed $\mathrm{Mg}$ and its alloys in relation to microstructural characteristics such as grain size and grain boundary character [6-8]. A consistent finding in these studies is that twinning activity is strongly correlated with grain boundary misorientation, with lower-angle grain boundaries encouraging the nucleation and growth of twins. It was also found that correlated twinning, where twins in neighboring grains meet at a boundary, is much more likely to occur at low angle grain boundaries. As all these studies were done post mortem at the resolution of EBSD, it could not be determined if twins meeting at the boundary arose due to simultaneous nucleation and growth into both grains or if it was an impingement and transmission or nucleation event as a single twin travelled across the boundary. Similar studies in $\mathrm{Zr}$ also showed a correlation between misorientation angle and propensity for twinning, though not as strongly as that seen in the Mg studies [9].

Bieler and colleagues have published a number of studies investigating individual instances of $\{1012\}$ twin interactions with grain boundaries in $\alpha$-Ti using EBSD analysis and in situ synchrotron characterization [10-12]. They found that when a grain is surrounded by other grains that are unfavorably oriented for slip, twin nucleation was dictated by the resolved shear stress. In contrast, when a grain was surrounded by other grains that are favorably oriented for slip, twin nucleation was initiated by dislocation pileups at the grain boundary. The nature of the twin plane and shear vector could be predicted based on maximizing the $m$ ' factor, which is given as [13]:

$m^{\prime}=\cos \kappa \cos \psi$

where $\kappa$ is the angle between slip plane normal of the incoming dislocation system and the twin plane normal and $\psi$ is the angle between shear/Burgers vectors of the two systems on either side of a grain boundary.

In the current study, twining behavior, with an emphasis on twin interactions with grain boundaries, are investigated in pure polycrystalline Re samples deformed under uniaxial compression. Multiple interactions were investigated using EBSD, after which TEM analysis was applied to investigate the interactions at higher resolution.

\section{Experimental methods}

99.99\% pure Re was supplied by Rhenium Alloys, Inc. in the form of a $3 \mathrm{~mm}$ diameter rod. $4 \mathrm{~mm}$ tall compression samples were cut from the rod using electrical discharge machining (EDM). The samples were then annealed for 20 hours at $1100^{\circ} \mathrm{C}$ in a $50 \%$ hydrogen, $50 \%$ argon environment. For digital image correlation (DIC) purposes, a speckle pattern was created on the sample surfaces by first spray painting them white, and then lightly spraying them with black spray paint (Fig. 1b). Two samples were strained in compression using a MTS Criterion Model 43 testing machine at a nominal strain rate of $10^{-3} \mathrm{~s}^{-1}$. The compression was recorded for DIC analysis using a 1280x1024-pixel Dino-Lite camera at a frame rate of 15 frames per second. DIC analysis of the images was performed in Matlab (analysis details can be found in [14]). 
Samples were prepared for TEM and EBSD analysis using EDM to cut two disks from the center of each compressed rod with an approximate thickness of $150 \mu \mathrm{m}$. Polishing of the sample surface was performed using a twin jet polisher with a $78 \%$ ethanol, $11 \%$ butoxyethanol, $11 \%$ perchloric electrolyte maintained at a temperature of $-10^{\circ} \mathrm{C}$. As needed, thinning to electron transparency was achieved using a PIPS II ion mill with a final accelerating voltage of $0.5 \mathrm{keV}$. Orientation-dependent etching rates resulted in ridging at twin and grain boundaries, which facilitated site-specific focused ion beam (FIB) lift-out samples to be machined from locations of interest. The post-FIB amorphous layer was removed using Ar-ion milling in a FEI NanoMill operated at $900 \mathrm{eV}$.

TEM analysis was performed using a JEOL 3010 TEM operated at $300 \mathrm{kV}$ as well as in a FEI Titan scanning (S)TEM also operated at $300 \mathrm{kV}$ operated in STEM mode. A camera length of $300 \mathrm{~mm}$ was used for diffraction contrast imaging. EBSD scans were taken using an accelerating voltage of $20 \mathrm{keV}$ over $70 \times 70 \mu \mathrm{m}$ regions at a $100 \mathrm{~nm}$ step size. No cleanup of the data was done except for the removal of points with a low confidence index value. The large grain-to-twin size ratio required a small step size be used in relation to the grain size, limiting the size of the scans acquired and reducing the statistical significance of the results.

\section{Results and Discussion}

The engineering stress/engineering strain curve for the compression tests is shown in Figure 1. The samples showed high rates of work hardening, similar to what has been reported previously $[2,4]$.

Inverse pole figure (IPF) maps created from the EBSD data and an IPF of the annealed Re are shown in Figure 2. Areas of poor indexing, represented as black pixels in the maps, are present. These were caused by differential etching rates dependent on crystal orientation leading to significant ridging occurring at grain boundaries and shadowing in the EBSD patterns. The decreased indexing rate did not adversely affect the analysis of the scans. The undeformed matrix is composed primarily of grains with the c-axis oriented perpendicular to the surface normal and an average grain size of $30 \mu \mathrm{m}$. No twins were seen in the pre-deformation microstructure and the uniform orientations across the grain interiors suggests that there are few dislocations present. As the deformation progresses, the microstructure becomes dominated by deformation twins with the twinned area of the microstructure increasing from $12.0 \%$ of the total area after compression to $5 \%$ strain (Fig. 1b) to $19.4 \%$ of the total area after compression to $8 \%$ strain (Fig. 1c). These twins were all of the same type, $\{1121\}\langle 1126\rangle$, and had an average width of $700 \mathrm{~nm}$. Multiple locations are evident in the scans where twins meet at the grain boundaries. At other locations, the twins impinge on only one side of the boundary with no correlated twin present in the neighboring grain. To examine the twin-grain boundary interactions in more depth, nine areas of interest were investigated, highlighted by boxes in Figure 2 and enlarged in Figure 3.

Of the nine highlighted interactions, six $(1,2,3,4,7,9)$ involved twins aligning exactly at the boundary while the other three $(5,6,8)$ either had twin activity only on one side of the 
boundary or the twins did not align at the boundary. The taper of some of the twins, specifically ' 4 ' and '7,' suggests a directionality to the interaction. That is, it appears that the twin boundary interaction is either an impingement and nucleation event or a direct transmission event rather than simultaneous nucleation of two twins at the boundary. The nine interactions were analyzed in terms of the grain boundary misorientation angle and the $m$ ' factor, described in equation 1. $m^{\prime}$ was calculated for all available $\{1121\}<1126\rangle$ twinning systems on one side of the boundary in relation to the twin impinging on the boundary plane. Table 1 summarizes the results of this analysis. In the case of twin transmission, the $m$ ' factor is calculated for the observed twin systems. When the twin does not transmit across the boundary, the maximum $m$ ' value is given by taking into account all of the available twin systems in the neighboring grain. The Schmid factor, calculated by considering only the macroscopically applied stress, was also taken into consideration but had no correlation to the observed twinning activity and so is not included in the table. This is likely due to the complex stress states that exist in the interior of polycrystalline materials.

Table 1. Analysis of twin/grain boundary interactions. Twin system number corresponds to labelling in Fig. 3.

\begin{tabular}{|c|c|c|c|c|c|c|c|c|c|c|}
\hline $\begin{array}{c}\text { Region of } \\
\text { interest }\end{array}$ & 1 & \multicolumn{2}{|c|}{$2^{1}$} & 3 & 4 & 5 & 6 & 7 & 8 & 9 \\
\hline $\begin{array}{c}\text { Boundary } \\
\text { misorientation }\end{array}$ & $15^{\circ}$ & \multicolumn{2}{|c|}{$23^{\circ}$} & $13^{\circ}$ & $18^{\circ}$ & $36^{\circ}$ & $72^{\circ}$ & $12^{\circ}$ & $57^{\circ}$ & $10^{\circ}$ \\
\hline$m^{\prime}$ & 0.94 & 0.91 & 0.91 & 0.95 & 0.95 & 0.97 & 0.19 & 0.96 & 0.54 & 0.97 \\
\hline $\begin{array}{c}\text { Transmits } \\
(\mathrm{Y} / \mathrm{N})\end{array}$ & $\mathrm{Y}$ & $\mathrm{Y}$ & $\mathrm{Y}$ & $\mathrm{Y}$ & $\mathrm{Y}$ & $\mathrm{N}$ & $\mathrm{N}$ & $\mathrm{Y}$ & $\mathrm{N}$ & $\mathrm{Y}$ \\
\hline
\end{tabular}

${ }^{1}$ Interaction 2 involves two twins on each side of the boundary

In all of the cases analyzed, the only consistent predictor of twin transmission was the misorientation angle across the grain boundary. Those boundaries with a misorientation angle at $23^{\circ}$ and below allowed transmission, while those with higher angles acted as barriers to twin propagation. All of the cases of twin transmission had a high $m$ ' value, suggesting this can be used as a predictive measure of which twinning system will activate at a grain boundary. However, as is seen in the case of interaction 5, a high $m$ ' value is not sufficient to guarantee twin transmission across a boundary. A higher misorientation angle will still result in the inability of the twin to transmit across the boundary.

The analysis of the interactions in regions 1,2, and 7 is shown in more detail in Figure 4 in which $m^{\prime}, \cos \kappa$, and $\cos \psi$ are plotted considering all available twin systems in relation to the impinging twin at the boundary. This analysis assumes a twin propagation direction, indicated in Figure 2. One can predict from Figure 4 which twinning system is activated considering only $m$ ' or $\cos \psi$, which are maximized in both interactions. Consideration of $\cos \kappa$ leads to inconsistent results as it is large for most of the available twinning systems, but maximized only in the twin 1 and twin 2 interactions. In the twin 7 interaction, emission of the ( 2 
111)[2116] twin would have resulted in a slightly higher value of $\cos \kappa$. Furthermore, the difference between the values of $\cos \kappa$ for different interactions are sufficiently close to be considered negligible. In other words, the angle between the incoming and outgoing shear vectors associated with the impinging twin is minimized, but alignment of the twin planes themselves does not appear to be as important. This holds true for all twin interaction events in this study; only $\cos \psi$ and $m$ ' were consistently maximized in all the interactions, suggesting that the primary influence is the minimization of the residual strain in the boundary. This is similar to what has been seen in dislocation/grain boundary interactions where the primary factor governing the interaction is the magnitude of the Burgers vector of the residual grain boundary dislocation [15-17].

Selected twin transmission events across grain boundaries were characterized in greater detail using TEM analysis. Figure 5a shows a twin interaction constructed from two different weak-beam dark field micrographs of the same area taken under different diffraction conditions (Fig. 5b-c). The imaged region consists of five twins, labeled 1-5 in the figure. Three twins in grain 1 are seen impinging on the boundary as well as two twins in grain 2 . Twin 3 and twin 4 appear to be continuous across the boundary. That is, the impingement point of the incoming twin and the nucleation point of the emitted twin correspond exactly. The relative widths of the two twins, with the emitted twin tapering to a point, suggest a directionality to the interaction. This implies that the impingement of one twin initiated the nucleation and growth of a twin in the neighboring grain or that the twin transmitted directly across the boundary. No dislocations are present in the image, suggesting that the plasticity is accommodated primarily through twinning mechanisms and that the twin nucleation in this instance is not instigated by a dislocation pile up. The misorientation angle across the grain boundary was found using diffraction analysis to be approximately $17^{\circ}$, below the threshold identified by the EBSD analysis to allow twin transmission.

A second twin/grain boundary interaction, this time captured in a FIB lift-out sample, is shown in Figure 6. The figure was taken using annular dark field STEM. A 330 nm-thick twin, labeled twin 1, is seen impinging on the boundary. The contrast in the twin interior suggests that there may be two secondary twins that have formed as well. The boundaries align exactly with twin 4 in the neighboring grain. There are also two much smaller twins, twin 2 and twin 3 , impinging on the grain boundary near the same spot. These could have nucleated later in the interaction to relieve built up strain energy in the boundary associated with the primary twin interaction. This would require in situ observations to confirm. A significant number of scattered dislocation are seen distributed throughout the grain and in the twin interiors, though there are no apparent pileups formed at either the grain or twin boundaries. EBSD analysis of the interaction showed the grain boundary to have a misorientation angle of $25^{\circ}$ and twins 1 and 4 to be $\{112$ $1\}<1126>$-type. Similar to what was seen in the EBSD results, $m$ ' was maximized in the interaction. The EBSD resolution was insufficient to characterize twins 2 and 3.

A FIB lift-out sample was also milled from a region where the twins did not transmit across the grain boundary. Figure 7 shows a triple junction where twins from Grain 1 and Grain 2 impinge on, but do not transmit across, the boundary between Grain 3. EBSD showed the 
misorientation between Grains 1 and 3 to be $35^{\circ}$; EBSD patterns from Grain 2 could not be indexed. In the figure, twins from Grains 1 and 2 are seen impinging on the grain boundary. No twin activity is seen in Grain 3. Secondary twins, emitted from the boundary, are also seen in Grain 1 at the interface of Grain 3. The dislocation density in Grain 3 is significantly higher than what is seen in Figures 5 and 6, suggesting that the strain at the boundary is mediated through dislocation plasticity as opposed to twinning.

\section{Discussion}

Similar to previous studies $[1,2]$, the work here has shown that $\{1121\}$ twinning is a dominant deformation mode in Re. The dominant grain orientations, with the [0001] oriented perpendicular to the compression direction, would also encourage the observed twinning behavior. This extensive twinning is likely responsible for much of rhenium's ductility, as well as a large contributing factor to its high work hardening rate as the twin planes create barriers to further dislocation and twinning-based plasticity. Many of the previous studies of Re have been limited to deformation of single crystals $[1,5]$. This study has shown the important role of grain boundaries in relation to twin deformation, with high angle grain boundaries providing much stronger barriers to twin propagation than low angle grain boundaries.

Previous studies on the statistical behavior of \{1012\}-type twins in Mg and $\mathrm{Zr}$ have shown that twinning activity can be correlated to the grain boundary rotation, with smaller misorientation angles across grain boundaries correlating with increased twinning activity and a higher number of aligned twins at the boundaries [6,9]. This work expands on those studies, showing that similar correlations can be made with $\{1121\}$ twins. Additionally, TEM results strongly suggest that the twin alignment at the boundary is due to twin propagation and transmission across the boundary as opposed to a simultaneous nucleation event. These twinning transmission events were limited to grain boundaries with misorientation angles at or below $25^{\circ}$, regardless of twin plane or shear vector alignment across the two crystal orientations.

With the information that twinning is a transmission event, these twin/grain boundary interactions can be treated in an analogous manner to dislocation/grain boundary interactions. Lee et al. have shown that for FCC materials [15], and more recent studies have corroborated in other crystal systems [16-18], that dislocation transmission across a grain boundary is dictated primarily by minimization of the Burgers vector of the residual grain boundary dislocation [1618]. The resolved shear stress was found to play a secondary role important primarily at the beginning of an interaction. Alignment of the slip planes of the incoming and outgoing dislocation systems was also considered and was found to be important only in cases of direct transmission where the slip planes must align in the grain boundary exactly. In considering twin interactions with grain boundaries in the present work, it was found that alignment of the shear vectors of the incoming and outgoing twins is the primary factor influencing the interaction. That is, for all the characterized twin transmission events, the emitted twin could be predicted knowing only the shear vector of the incoming twin and the character of the grain boundary. Alternatively, $m$ ', which also takes into account alignment of the twin planes, can be used as a 
predictive measure dictating the relationship of twin transmission. The alignment of the twin planes alone, however, was not consistent among the observed interactions. This result can be understood by considering the elastic strain energy stored at the boundary. Any misalignment of the shear vectors of the incoming and outgoing twins from the boundary results in an increase in the elastic strain energy, creating an energy barrier against further transfer of strain across the boundary. This suggests that the twin transfer across the grain boundary is likely an impingement and nucleation event as twin plane alignment would be a more important factor in dictating direct transmission.

The analogy between dislocation/grain boundary interactions and twin/grain boundary interactions breaks down when considering the influence of grain boundary misorientation on the likelihood of a transmission event occurring. Wang et al. suggested, based on atomistic simulations of symmetric tilt grain boundaries, that low angle grain boundaries promote twinning due to their intrinsic dislocation structure [19]. They showed that in $\mathrm{Mg}$, grain boundaries with misorientation angles below $13.2^{\circ}$ can be described as an array of discrete dislocations. Misorientations in the range of $13.2-28^{\circ}$ cause the dislocation spacing to decrease to the point that they begin to lose their individual character. Grain boundaries with misorientation angles above $28^{\circ}$ undergo a further transformation where they are composed of low sigma, coherent boundaries such as twin boundaries with dislocation arrays to accommodate any deviation from coherency. This variation in grain boundary structure was found to be the main cause for a decrease in twin nucleation events at high angle grain boundaries and agrees well with the cut off angle of $25^{\circ}$ for twin transmission observed for Re in our study. At higher misorientation angles, TEM imaging of the deformed microstructure suggests that strain transfer across grain boundaries is accomplished via slip-twin interactions, similar to what was suggested by Bieler $e t$ al. during straining of $\alpha-\mathrm{Ti}[10]$.

\section{Conclusion}

Twin interactions with grain boundaries in pure Re have been studied using EBSD and TEM characterization. Images of the twin interactions suggest that impinging twins transmit across the grain boundary, likely via an impingement and nucleation event. It was found that grain boundaries with a misorientation angle at or below $25^{\circ}$ allowed twin transmission while those with higher misorientation angles were strong barriers to twin propagation. Alignment of the shear vector associated with the incoming and outgoing twinning systems was determined to be the governing factor dictating which twinning system nucleated at a grain boundary.

\section{Acknowledgements:}

We gratefully acknowledge funding from the US Office of Naval Research under Grant No. N00014-11-1-0886. Portions of this work were performed as a user project at the National Center for Electron Microscopy at Lawrence Berkeley National Laboratory, which is supported 
by the U.S. Department of Energy under Contract \# DE-AC02-05CH11231. EBSD work was done at the EDAX-TSL facilities in Draper, UT.

1. Kopetskii, C.V., M.M. Myshlyaev, N.I. Novokhatskaya, and V.A. Yukhanov, Fizika Metallov i Metallovedenie, 1975; 39(1): 165-74.

2. Churchman, A.T., Metallurgical Society of American Institute of Mining, Metallurgical and Petroleum Engineers -- Transactions, 1960; 218(2): 262-267.

3. Bryskin, B.D. and F.C. Danek, JOM Journal of the Minerals Metals and Materials Society, 1991; 43(7): 24-28.

4. $\quad$ Bryskin, B.D., AIP Conference Proceedings, 1992; 246: 278-91.

5. Churchman, A.T., Proceedings of the Royal Society of London, Series A, 1954; 226: 216-226.

6. Beyerlein, I.J., L. Capolungo, P.E. Marshall, R.J. McCabe, and C.N. Tome, Philosophical Magazine, 2010; 90(16): 2161-90.

7. Tome, C.N., I.J. Beyerlein, J. Wang, and R.J. McCabe, JOM, 2011; 63(3): 19-23.

8. Beyerlein, I.J., R.J. McCabe, and C.N. Tome, Journal of the Mechanics and Physics of Solids, 2011; 59(5): 988-1003.

9. Capolungo, L., P.E. Marshall, R.J. McCabe, I.J. Beyerlein, and C.N. Tome, Acta Materialia, 2009; 57(20): 6047-56.

10. Bieler, T.R., L. Wang, A.J. Beaudoin, P. Kenesei, and U. Lienert, Metallurgical and Materials Transactions A, 2014; 45: 109-122.

11. Wang, L., Y. Yang, P. Eisenlohr, T.R. Bieler, M.A. Crimp, and D.E. Mason, Metallurgical and Materials Transactions A, 2010; 41(2): 421-430.

12. Wang, L., P. Eisenlohr, Y. Yang, T.R. Bieler, and M.A. Crimp, Scripta Materialia, 2010; 63(8): 827-830.

13. Luster, J. and M.A. Morris, Metallurgical and Materials Transactions A, 1995; 26 A(7): 1745-1756.

14. Jones, E.M.C., M.N. Silberstein, S.R. White, and N.R. Sottos, Experimental Mechanics, 2014.

15. Lee, T.C., I.M. Robertson, and H.K. Birnbaum, Philosophical Magazine A, 1990; 62(1): 131-53.

16. Kacher, J. and I.M. Robertson, Acta Materialia, 2012; 60(19): 6657-72.

17. Kacher, J. and I.M. Robertson, Philosophical Magazine, 2014; 94(8): 814-829.

18. Gemperle, A., J. Gemperlova, and N. Zarubova, Materials Science and Engineering A, 2004; 387-389: 46-50.

19. Wang, J., R.G. Hoagland, J.P. Hirth, L. Capolungo, I.J. Beyerlein, and C.N. Tome, Scripta Materialia, 2009; 61(9): 903-6.

\section{Figures}

Figure 1. a) Engineering stress/engineering strain of the compressed Re samples. Curves are labelled for reference in the text. b) speckled sample used in compression test. 
Figure 2. IPF map and 0001 IPF of an annealed Re sample (a-b), IPF map of sample 1 after $5 \%$ compression deformation (c), and IPF map of sample 2 after $8 \%$ compression deformation (d). IPF maximum value is 3 times random. Boxed areas are examples of twin/grain boundary interactions and are enlarged in Fig. 3 for more in depth analysis. The color map legend is included in (d).

Figure 3. Enlarged regions corresponding to the boxed regions in Fig. 2. Arrows indicate suggested twin propagation direction. Wire lattice images are included in each frame showing the orientation of the parent grain.

Figure 4. $m$ ', $\cos (\kappa)$, and $\cos (\psi)$ values for the twin interactions in regions 1 (a), 2 (b), and 7 (c) for all available twinning systems at the emission side of the grain boundary. The observed emitted twin system is indicated by an arrow for each interaction.

Figure 5. Twin/grain boundary interaction observed in a jet-polished Re sample postdeformation. a) is a merged weak beam dark field image compiled of (b) and (c). Diffraction patterns associated with (b) and (c) are given in the image insets. The diffraction pattern in the inset in (a) is taken from the boundary at a zone axis showing the crystal rotation. The assumed twin propagation direction is indicated by an arrow. All other labelling is for reference in the text.

Figure 6. Annular dark field STEM image of a twin interaction captured in a FIB lift-out sample. (b) is image of boxed region in (a) taken at higher magnification. Labelling is for reference in the text.

Figure 7. Annular dark field STEM image of a twin interaction captured in a FIB lift-out sample. (a) was constructed by combining (b) and (c). Grain boundaries are marked by a dotted line and arrows indicate assumed twin propagation direction. Grain labelling is for reference in the text. 

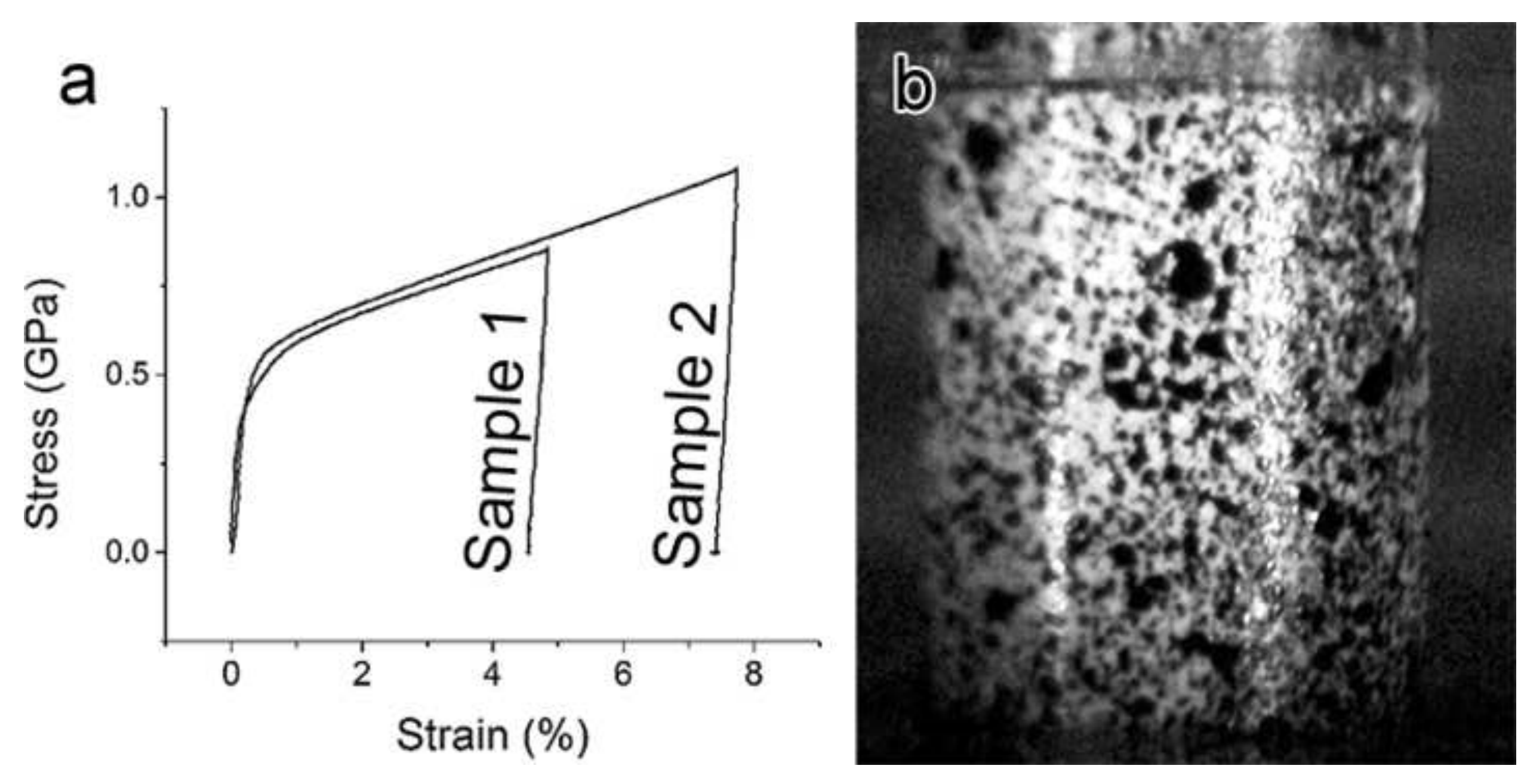


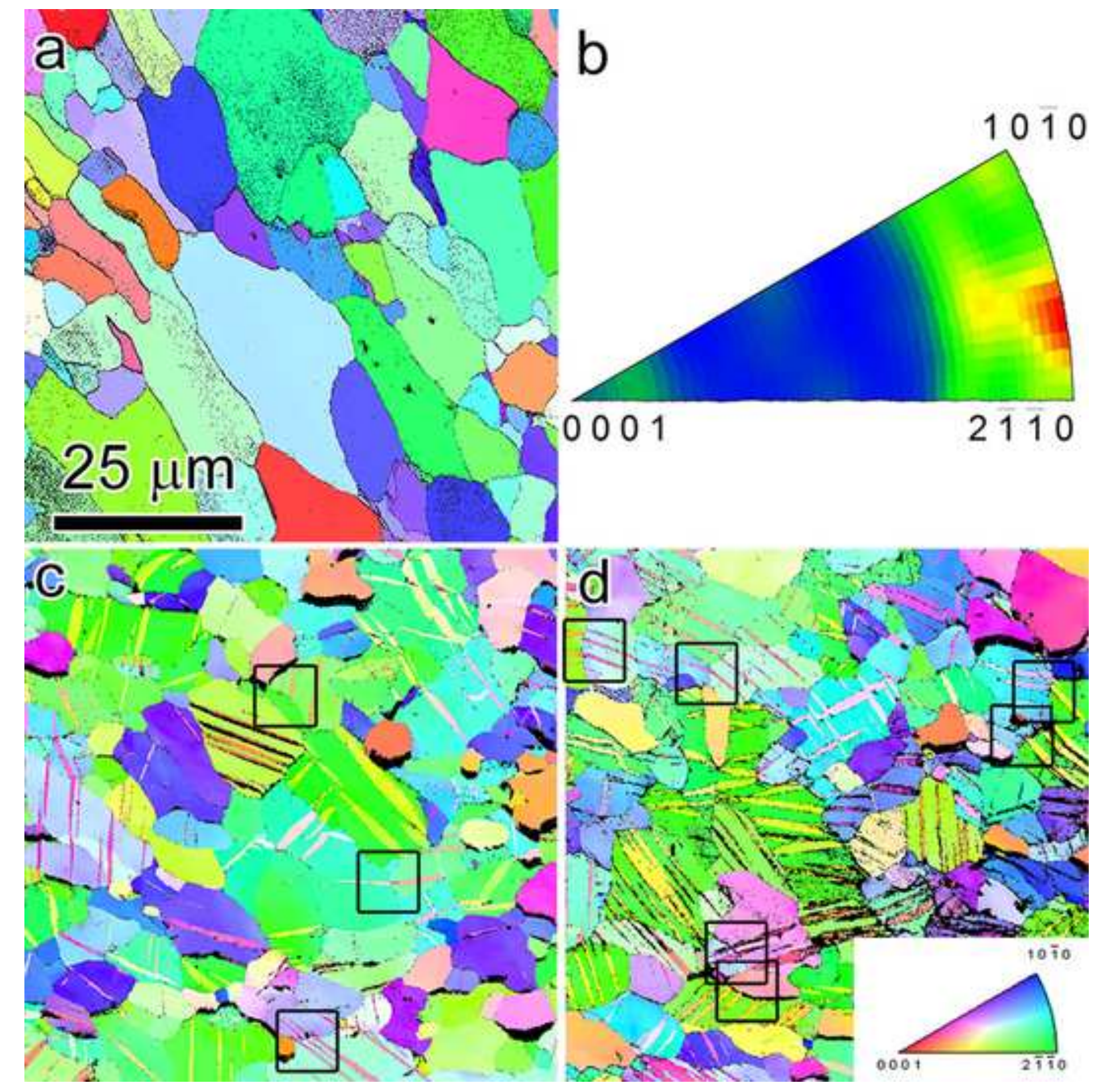



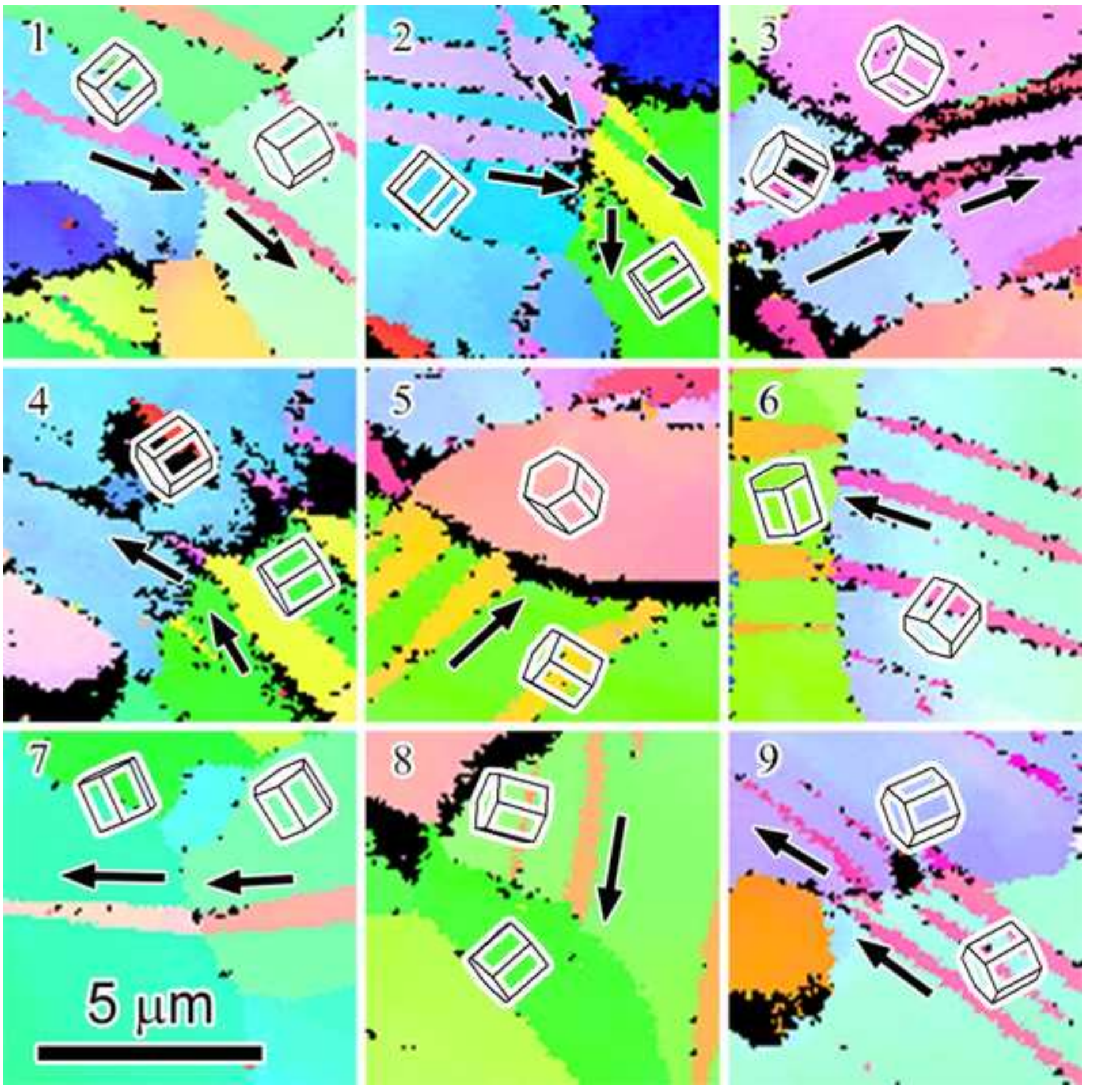


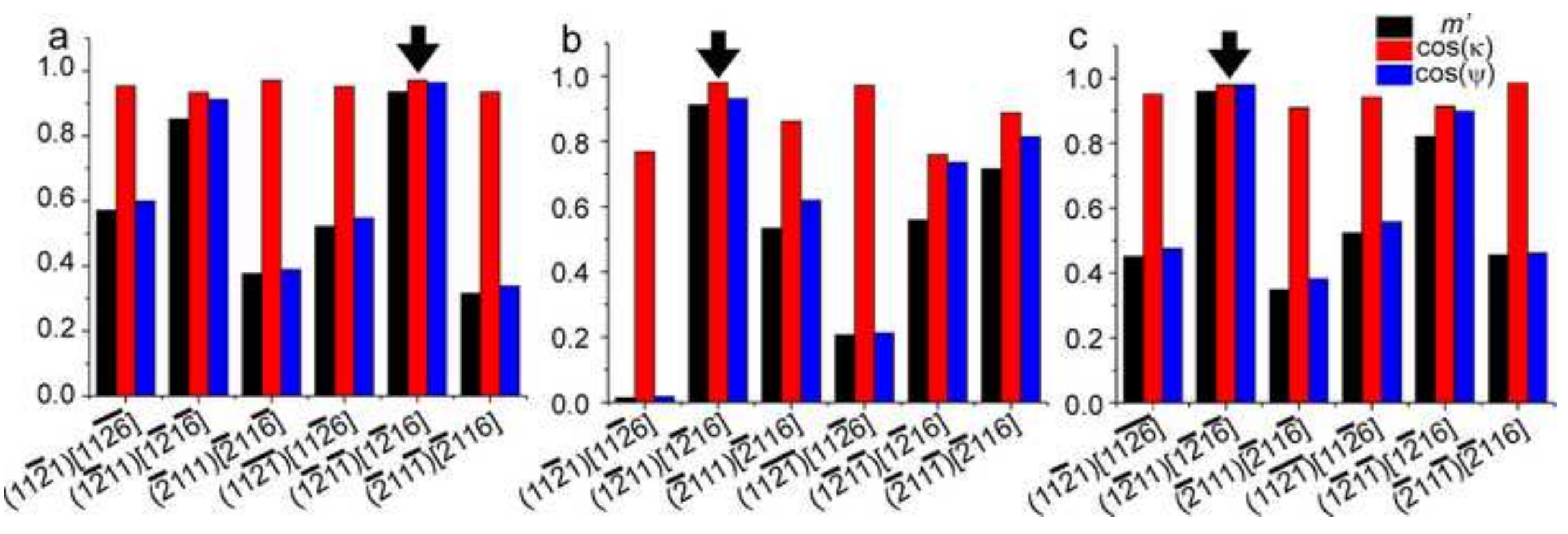




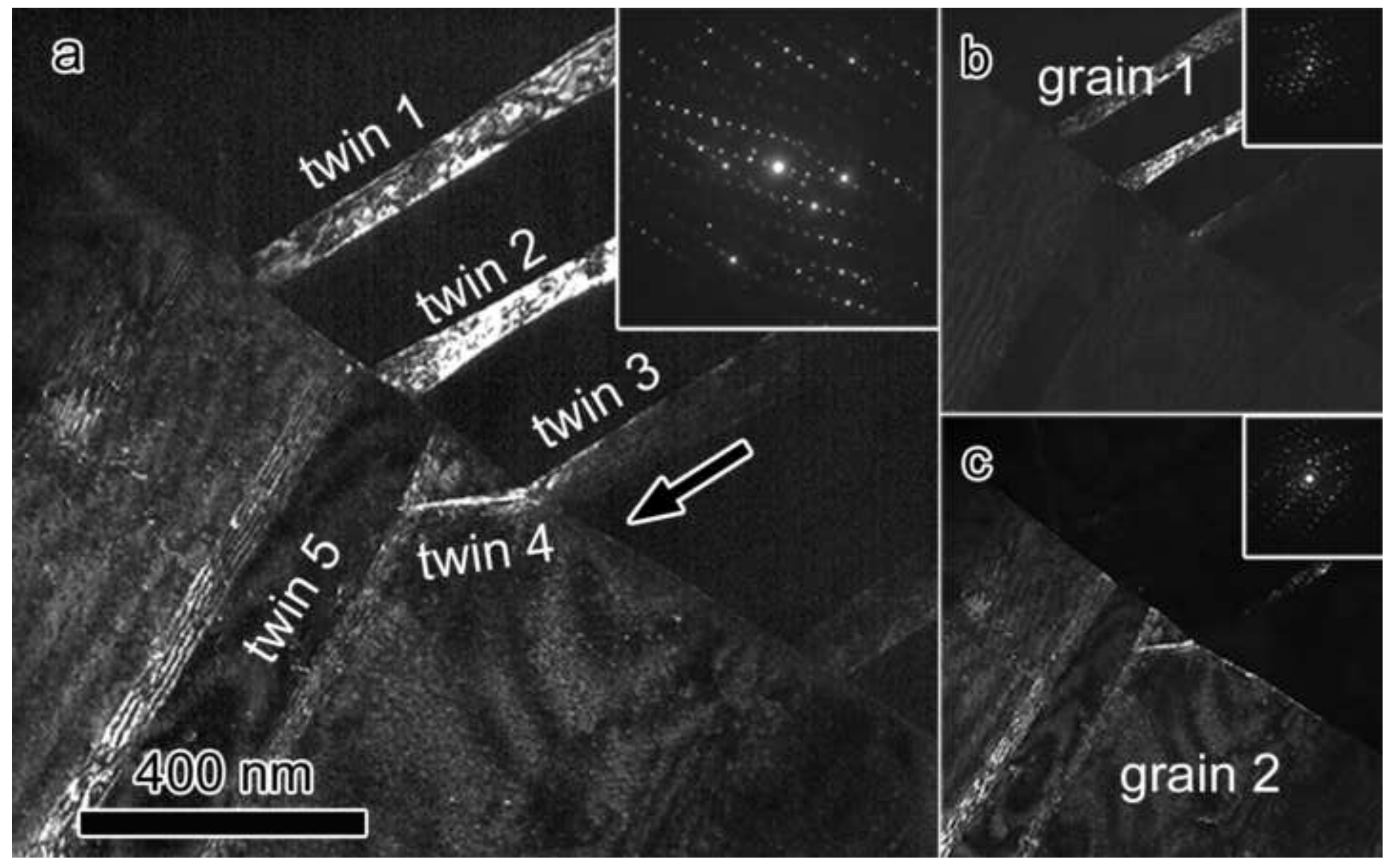

a 

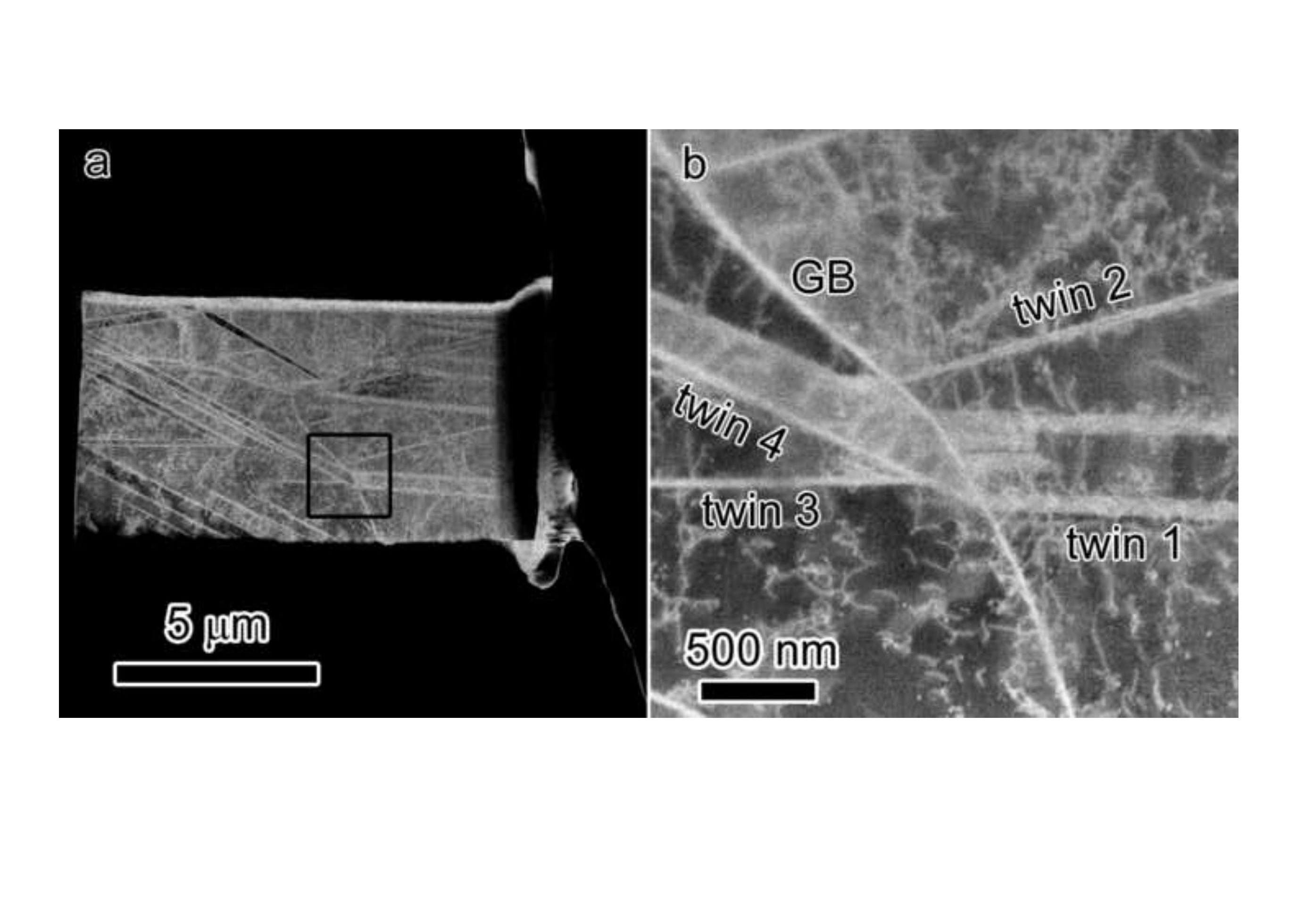

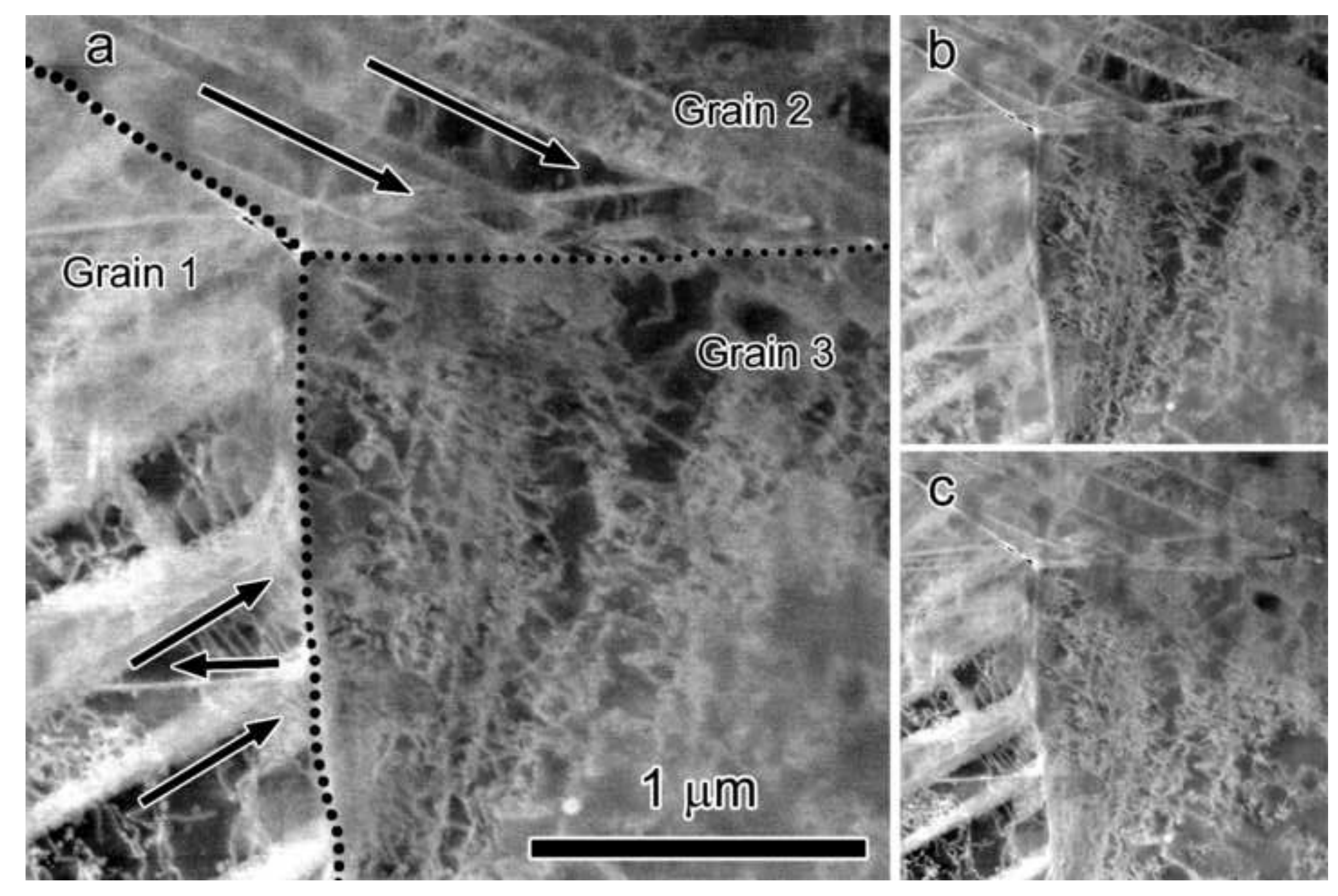